\title{
Testing sphericity and intraclass covariance structures under a Growth Curve model in high dimension
}

Muni S. Srivastava and Martin Ohlson

The self-archived postprint version of this journal article is available at Linköping University Institutional Repository (DiVA):

http:// urn.kb.se/ resolve?urn=urn:nbn:se:liu:diva-152116

N.B.: When citing this work, cite the original publication.

This is an electronic version of an article published in:

Srivastava, M. S., Ohlson, M., (2017), Testing sphericity and intraclass covariance structures under a Growth Curve model in high dimension, Communications in statistics. Simulation and computation, 46(7), 5740-5751. https:// doi.org/ 10.1080/03610918.2016.1175623

Original publication available at:

https:/ / doi.org/ 10.1080/03610918.2016.1175623

Copyright: Taylor \& Francis (STM, Behavioural Science and Public Health Titles)

http:// www.tandf.co.uk/journals/ default.asp 


\title{
Testing Sphericity and Intraclass Covariance Structures under a Growth Curve Model in High Dimension
}

\author{
Muni S. Srivastava \\ Department of Statistics, University of Toronto \\ Martin Singull* \\ Department of Mathematics, Linköping University
}

\begin{abstract}
In this paper we consider the problem of testing (a) sphericity and (b) intraclass covariance structure under a Growth Curve model. The maximum likelihood estimator (MLE) for the mean in a Growth Curve model is a weighted estimator with the inverse of the sample covariance matrix which is unstable for large $p$ close to $N$ and singular for $p$ larger than $N$. The MLE for the covariance matrix is based on the MLE for the mean, which can be very poor for $p$ close to $N$. For both structures (a) and (b), we modify the MLE for the mean to an unweighted estimator and based on this estimator we propose a new estimator for the covariance matrix. This new estimator leads to new tests for (a) and (b). We also propose two other tests for each structure, which are just based on the sample covariance matrix.

To compare the performance of all four tests we compute for each structure (a) and (b) the attained significance level and the empirical power. We show that one of the tests based on the sample covariance matrix is better than the likelihood ratio test based on the MLE.
\end{abstract}

Keyword: Growth Curve model; GMANOVA; Sphericity; Intraclass covariance structure; Hypothesis testing; Asymptotic distribution; Power comparison; High dimension.

*Corresponding author: martin.singull@liu.se 


\section{Introduction}

In this paper we consider the problem of testing sphericity and intraclass covariance structure under a Growth Curve model. The general multivariate linear model, the so called Growth Curve Model, was introduced by Potthoff and Roy (1964) when the $p \times N$ observation matrix is normally distributed with an unknown covariance matrix. The Growth Curve model belongs to the curved exponential family, since it is a generalized multivariate analysis of variance model (GMANOVA). The mean structure for the Growth Curve model is bilinear, i.e., two design matrices are involved, in contrary to the ordinary multivariate analysis of variance (MANOVA) model where the mean is linear. For more details about the Growth Curve model see e.g., Kollo and von Rosen (2005); Kshirsagar and Smith (1995); Srivastava and Khatri (1979); Srivastava and von Rosen (1999).

For the Growth Curve model, when no assumption about the covariance matrix was made, Potthoff and Roy (1964) originally derived a class of weighted estimators for the mean parameter matrix. Khatri (1966) extended this result and showed that the maximum likelihood estimator is also a weighted estimator. Under a certain covariance structure, Rao (1967) and Reinsel (1982) have shown that the unweighted estimator is also the maximum likelihood estimator. Furthermore, Chinchilli and Walter Jr (1984) have derived the likelihood ratio test for this type of covariance matrix.

In the MANOVA model, when dealing with measurements on $k$ equivalent psychological tests, Wilks (1946) was one of the first to consider patterned covariance matrices. A covariance matrix with equal diagonal elements and equal off-diagonal elements, i.e., a so-called intraclass covariance structure, was studied. For the Growth Curve model Khatri (1973) derived the likelihood ratio test for the intraclass covariance structure and Lee (1988) considered prediction for the model. Recently, there have again been some focus on the intraclass covariance structure for the Growth Curve model. Žežula (2006) derived some simple explicit estimators of the variance and the correlation given the intraclass covariance structure. The estimators were based on the unbiased estimating equations. Also based on the unbiased estimating equations, using an orthogonal decomposition, Ye and Wang (2009) and Klein and Žežula (2010) derived estimators for the intraclass covariance structure.

Closely connected to the intraclass covariance structure, with positive covariances, is the random effects covariance structure studied by Rao (1965, 1975), Reinsel (1982, 1984), Ware (1985) and recently by Srivastava and Singull (2012) for a parallel profile model. The random-effect covariance structure has also been considered for the mixed MANOVA-GMANOVA models and the Extended Growth Curve models, see e.g., Yokoyama (1995, 1996, 
1997).

The organization of the paper is as follows. In Section 2, we define the Growth Curve model and give the two jointly sufficient statistics. The MLE and an unweighted estimator are given in Section 3, where also a comparison of the two estimators is discussed. In Section 4 we give four tests for sphericity, the LRT and three other tests based on the unweighted estimator and the sum of squares, and similar in Section 5 for the intraclass covariance structure. In Section 6 the attained significance levels and the empirical powers are compared. The paper concludes in Section 7 .

\section{Model}

In this section, some general ideas of how to estimate parameters in the Growth Curve model will be presented. The Growth Curve model introduced by Potthoff and Roy (1964) and Rao (1965) is defined as follows.

Definition Let $\boldsymbol{X}: p \times N$ and $\boldsymbol{\xi}: q \times m$ be the observation and parameter matrices, respectively, and let $\boldsymbol{B}: p \times q$ and $\boldsymbol{A}: m \times N$ be the within and between individual design matrices, respectively. Suppose that $q \leq p$ and $r+p \leq N$, where $r=\operatorname{rank}(\boldsymbol{A})$. The Growth Curve model is given by

$$
\boldsymbol{X}=\boldsymbol{B} \boldsymbol{\xi} \boldsymbol{A}+\boldsymbol{\varepsilon},
$$

where the columns of $\varepsilon$ are assumed to be independently $p$-variate normally distributed with mean zero and an unknown positive definite covariance matrix $\Sigma$, i.e.,

$$
\varepsilon \sim N_{p, N}\left(\mathbf{0}, \boldsymbol{\Sigma}, \boldsymbol{I}_{N}\right),
$$

where $N_{p, N}(\bullet, \bullet, \bullet)$ stands for the matrix normal distribution.

In this paper, for the sake of convenience and without loss of generality, we will assume that the design matrices $\boldsymbol{B}$ and $\boldsymbol{A}$ are of full rank, i.e., $\operatorname{rank}(\boldsymbol{B})=q \leq p$ and $r=\operatorname{rank}(\boldsymbol{A})=m \leq N$.

Our starting point for estimating parameters in the Growth Curve model are the two jointly sufficient statistics, the "mean" $\boldsymbol{X} \boldsymbol{A}^{\prime}\left(\boldsymbol{A} \boldsymbol{A}^{\prime}\right)^{-1} \boldsymbol{A}$ and the sum of squares matrix

$$
\boldsymbol{V}=\boldsymbol{X}\left(\boldsymbol{I}-\boldsymbol{A}^{\prime}\left(\boldsymbol{A} \boldsymbol{A}^{\prime}\right)^{-1} \boldsymbol{A}\right) \boldsymbol{X}^{\prime} .
$$

The distribution of the "mean" and the sum of squares matrix are given by

$$
\boldsymbol{X} \boldsymbol{A}^{\prime}\left(\boldsymbol{A} \boldsymbol{A}^{\prime}\right)^{-1} \boldsymbol{A} \sim N_{p, N}\left(\boldsymbol{B} \boldsymbol{\xi} \boldsymbol{A}, \boldsymbol{\Sigma}, \boldsymbol{A}^{\prime}\left(\boldsymbol{A} \boldsymbol{A}^{\prime}\right)^{-1} \boldsymbol{A}\right)
$$


and

$$
\boldsymbol{V} \sim W_{p}(\boldsymbol{\Sigma}, n)
$$

where $W_{p}(\bullet, \bullet)$ stands for the Wishart distribution with $n=N-m$ degrees of freedom. Observe that $\boldsymbol{V}$ is independent of the parameter $\boldsymbol{\xi}$.

\section{Estimators}

In this section we will give the MLE of the mean parameter $\boldsymbol{\xi}$ and the covariance matrix $\boldsymbol{\Sigma}$. We also give the unweighted estimator discussed by Srivastava and Singull (2015).

\subsection{MLEs of the parameters $\xi$ and $\Sigma$}

The MLEs of $\boldsymbol{\xi}$ and $\boldsymbol{\Sigma}$ were given by Khatri (1966). The MLE of $\boldsymbol{\xi}$ is given by

$$
\widehat{\boldsymbol{\xi}}_{M L E}=\left(\boldsymbol{B}^{\prime} \boldsymbol{V}^{-1} \boldsymbol{B}\right)^{-1} \boldsymbol{B}^{\prime} \boldsymbol{V}^{-1} \boldsymbol{X} \boldsymbol{A}^{\prime}\left(\boldsymbol{A} \boldsymbol{A}^{\prime}\right)^{-1}
$$

and the MLE of $\boldsymbol{\Sigma}$ is

$$
\begin{aligned}
N \widehat{\boldsymbol{\Sigma}}_{M L E} & =\left(\boldsymbol{X}-\boldsymbol{B} \widehat{\boldsymbol{\xi}}_{M L E} \boldsymbol{A}\right)\left(\boldsymbol{X}-\boldsymbol{B} \widehat{\boldsymbol{\xi}}_{M L E} \boldsymbol{A}\right)^{\prime} \\
& =\boldsymbol{V}+\left(\boldsymbol{I}_{p}-\boldsymbol{P}_{\boldsymbol{B}}^{\boldsymbol{V}}\right) \boldsymbol{V}_{1}\left(\boldsymbol{I}_{p}-\boldsymbol{P}_{\boldsymbol{B}}^{\boldsymbol{V}}\right)^{\prime}
\end{aligned}
$$

where

$$
\begin{aligned}
\boldsymbol{V} & =\boldsymbol{X}\left(\boldsymbol{I}_{N}-\boldsymbol{P}_{\boldsymbol{A}^{\prime}}\right) \boldsymbol{X}^{\prime}=\boldsymbol{X} \boldsymbol{X}^{\prime}-\boldsymbol{V}_{1}, \\
\boldsymbol{V}_{1} & =\boldsymbol{X} \boldsymbol{A}^{\prime}\left(\boldsymbol{A} \boldsymbol{A}^{\prime}\right)^{-1} \boldsymbol{A} \boldsymbol{X}^{\prime},
\end{aligned}
$$

and where the projections $\boldsymbol{P}_{\boldsymbol{A}^{\prime}}$ and $\boldsymbol{P}_{\boldsymbol{B}}^{\boldsymbol{V}}$ are defined as

$$
\begin{aligned}
\boldsymbol{P}_{\boldsymbol{A}^{\prime}} & =\boldsymbol{A}^{\prime}\left(\boldsymbol{A} \boldsymbol{A}^{\prime}\right)^{-1} \boldsymbol{A}, \\
\boldsymbol{P}_{\boldsymbol{B}}^{V} & =\boldsymbol{B}\left(\boldsymbol{B}^{\prime} \boldsymbol{V}^{-1} \boldsymbol{B}\right)^{-1} \boldsymbol{B}^{\prime} \boldsymbol{V}^{-1}
\end{aligned}
$$

with the notation $\boldsymbol{P}_{\boldsymbol{B}}^{\boldsymbol{I}}=\boldsymbol{P}_{\boldsymbol{B}}$. The mean and covariance matrix for the estimator $\widehat{\boldsymbol{\xi}}_{M L E}$ are given in Kollo and von Rosen (2005) as

$$
\begin{aligned}
\mathrm{E}\left(\widehat{\boldsymbol{\xi}}_{M L E}\right) & =\boldsymbol{\xi}, \\
\operatorname{cov}\left(\widehat{\boldsymbol{\xi}}_{M L E}\right) & =\frac{n-1}{n-1-(p-q)}\left(\boldsymbol{A} \boldsymbol{A}^{\prime}\right)^{-1} \otimes\left(\boldsymbol{B}^{\prime} \boldsymbol{\Sigma}^{-1} \boldsymbol{B}\right)^{-1},
\end{aligned}
$$


if $n-1-(p-q)>0$. Since $q \leq p$ we note that

$$
\frac{n-1}{n-1-(p-q)} \geq 1
$$

Furthermore, the expectation of the $\widehat{\boldsymbol{\Sigma}}_{M L E}$ is given by von Rosen (1991) as

$$
\mathrm{E}\left(\widehat{\boldsymbol{\Sigma}}_{M L E}\right)=\boldsymbol{\Sigma}-\frac{m}{N} \frac{N-m-2(p-q)-1}{N-m-(p-q)-1} \boldsymbol{B}\left(\boldsymbol{B}^{\prime} \boldsymbol{\Sigma}^{-1} \boldsymbol{B}\right)^{-1} \boldsymbol{B}^{\prime} .
$$

Clearly, the estimator $\widehat{\boldsymbol{\Sigma}}_{M L E}$ is not an unbiased estimator of $\boldsymbol{\Sigma}$. The bias depends on the design matrix $\boldsymbol{B}$ and thus it could be a significant bias for relatively small samples.

\subsection{Unweighted estimator of the parameters $\xi$ and $\Sigma$}

A natural alternative to the MLE would be an unweighted estimator of $\boldsymbol{\xi}$ which is discussed by Srivastava and Singull (2015) and is given by

$$
\widehat{\boldsymbol{\xi}}_{U W}=\left(\boldsymbol{B}^{\prime} \boldsymbol{B}\right)^{-1} \boldsymbol{B}^{\prime} \boldsymbol{X} \boldsymbol{A}^{\prime}\left(\boldsymbol{A} \boldsymbol{A}^{\prime}\right)^{-1} \text {. }
$$

This estimator is simpler than the MLE, given in (1), since we do not need to calculate the inverse $\boldsymbol{V}^{-1}$ of the sum of squares matrix, which may not be stable for $p$ close to $n, p<n$. The distribution of the unweighted estimator is given by

$$
\widehat{\boldsymbol{\xi}}_{U W} \sim N_{q, m}\left(\boldsymbol{\xi},\left(\boldsymbol{B}^{\prime} \boldsymbol{B}\right)^{-1} \boldsymbol{B}^{\prime} \boldsymbol{\Sigma} \boldsymbol{B}\left(\boldsymbol{B}^{\prime} \boldsymbol{B}\right)^{-1},\left(\boldsymbol{A} \boldsymbol{A}^{\prime}\right)^{-1}\right) .
$$

From above, we get

$$
\operatorname{cov}\left(\widehat{\boldsymbol{\xi}}_{U W}\right)=\left(\boldsymbol{A} \boldsymbol{A}^{\prime}\right)^{-1} \otimes\left(\boldsymbol{B}^{\prime} \boldsymbol{B}\right)^{-1} \boldsymbol{B}^{\prime} \boldsymbol{\Sigma} \boldsymbol{B}\left(\boldsymbol{B}^{\prime} \boldsymbol{B}\right)^{-1} .
$$

It has been shown by Rao (1967) (Lemma 2.c) that

$$
\left(\boldsymbol{B}^{\prime} \boldsymbol{\Sigma}^{-1} \boldsymbol{B}\right)^{-1} \leq\left(\boldsymbol{B}^{\prime} \boldsymbol{B}\right)^{-1} \boldsymbol{B}^{\prime} \boldsymbol{\Sigma} \boldsymbol{B}\left(\boldsymbol{B}^{\prime} \boldsymbol{B}\right)^{-1},
$$

with equality if and only if $\mathcal{C}\left(\boldsymbol{\Sigma}^{-1} \boldsymbol{B}\right)=\mathcal{C}(\boldsymbol{B})$. The inequality is with respect to the Loewner partial ordering, i.e., $\left(\boldsymbol{B}^{\prime} \boldsymbol{B}\right)^{-1} \boldsymbol{B}^{\prime} \boldsymbol{\Sigma} \boldsymbol{B}\left(\boldsymbol{B}^{\prime} \boldsymbol{B}\right)^{-1}-\left(\boldsymbol{B}^{\prime} \boldsymbol{\Sigma}^{-1} \boldsymbol{B}\right)^{-1}$ is nonnegative definite. But for large $p$ the expression in (7) could be much larger than one. Thus the superiority of one over the other will depend on the dimension $p$. If the dimension $p$ is large, the estimator given in (8) could be preferred.

It may be noted that the unweighted estimator $\widehat{\boldsymbol{\xi}}_{U W}$ in (8) is the MLE when $\mathcal{C}\left(\boldsymbol{\Sigma}^{-1} \boldsymbol{B}\right)=\mathcal{C}(\boldsymbol{B})$, for example when $\boldsymbol{\Sigma}=\sigma^{2} \boldsymbol{I}_{p}$. Hence, this is also 
true for Rao's simple structure $\boldsymbol{\Sigma}=\boldsymbol{B} \boldsymbol{\Gamma} \boldsymbol{B}^{\prime}+\boldsymbol{Z} \boldsymbol{\Theta} \boldsymbol{Z}^{\prime}$, where $\boldsymbol{\Gamma}$ and $\boldsymbol{\Theta}$ are unknown symmetric matrices and $\boldsymbol{Z}: p \times(p-m)$ is known with rank $p-m$ such that $\boldsymbol{B}^{\prime} \boldsymbol{Z}=\mathbf{0}$, first given by Rao (1967). Furthermore, for the special case, the intraclass covariance structure, $\boldsymbol{\Sigma}=\sigma^{2}\left((1-\rho) \boldsymbol{I}_{p}+\rho \mathbf{1 1}^{\prime}\right)$, where $-\frac{1}{p-1}<\rho<1$ and $\mathbf{1}$ is a vector of ones, and where the first column in $\boldsymbol{B}$ is a column of ones, as in many applications, the unweighted estimator $\widehat{\boldsymbol{\xi}}_{U W}$ in (8) is the MLE.

Following the usual method of obtaining an estimator of $\boldsymbol{\Sigma}$, we obtain an estimator of $\Sigma$ by using the estimator given in (8). This new estimator of $\Sigma$ is given by

$$
\begin{aligned}
N \widehat{\boldsymbol{\Sigma}}_{U W} & =\left(\boldsymbol{X}-\boldsymbol{B} \widehat{\boldsymbol{\xi}}_{U W} \boldsymbol{A}\right)\left(\boldsymbol{X}-\boldsymbol{B} \widehat{\boldsymbol{\xi}}_{U W} \boldsymbol{A}\right)^{\prime} \\
& =\boldsymbol{V}+\left(\boldsymbol{I}_{p}-\boldsymbol{P}_{\boldsymbol{B}}\right) \boldsymbol{V}_{1}\left(\boldsymbol{I}_{p}-\boldsymbol{P}_{\boldsymbol{B}}\right)^{\prime}
\end{aligned}
$$

Again the bias could be significant with

$$
\mathrm{E}\left(\widehat{\boldsymbol{\Sigma}}_{U W}\right)=\boldsymbol{\Sigma}+\frac{m}{N}\left(\boldsymbol{\Sigma}+\left(\boldsymbol{I}_{p}-\boldsymbol{P}_{\boldsymbol{B}}\right) \boldsymbol{\Sigma}\left(\boldsymbol{I}_{p}-\boldsymbol{P}_{\boldsymbol{B}}\right)^{\prime}\right) .
$$

The bias in estimating $\boldsymbol{\Sigma}$ either by the use of $\widehat{\boldsymbol{\xi}}_{M L E}$ give in (1) or $\widehat{\boldsymbol{\xi}}_{U W}$ given in (8), can be very large.

However, it is known that under normality, there exist an uniformly minimum variance unbiased invariant estimator for $\Sigma$ given by

$$
\widehat{\Sigma}=\frac{V}{n}
$$

where $n=N-m$, see Žežula (1993) for more details. Thus, for the inference on the covariance matrix $\boldsymbol{\Sigma}$, one should use the simple estimator given above in $(10)$.

\section{Testing Sphericity}

In this section we test the hypothesis about sphericity, i.e.,

$$
H_{1}: \boldsymbol{\Sigma}=\sigma^{2} \boldsymbol{I}_{p} \quad \text { vs. } \quad A_{1}: \boldsymbol{\Sigma}>0 .
$$

We will give four different test statistics with corresponding asymptotic null distributions. In Section 6 we will compare the performance of these four statistics. 


\subsection{Standard Likelihood Ratio Test for sphericity}

The standard LRT for testing the hypothesis $H_{1}$ is based on the MLEs, (1) and (2). This test should not be preferred since it is based on a biased estimator of $\boldsymbol{\Sigma}$, and the bias depending on the design matrix $\boldsymbol{B}$. Anyway, the standard LRT as given in Khatri (1973) as

$$
\lambda_{1}=\frac{\left|\boldsymbol{V}+\left(\boldsymbol{I}-\boldsymbol{P}_{\boldsymbol{B}}^{\boldsymbol{V}}\right) \boldsymbol{V}_{1}\left(\boldsymbol{I}-\boldsymbol{P}_{\boldsymbol{B}}^{\boldsymbol{V}}\right)^{\prime}\right|}{\left(\frac{\operatorname{tr} \boldsymbol{V}+\operatorname{tr}\left(\boldsymbol{I}-\boldsymbol{P}_{\boldsymbol{B}}\right) \boldsymbol{V}_{1}}{p}\right)^{p}},
$$

where $\boldsymbol{V}, \boldsymbol{V}_{1}, \boldsymbol{P}_{\boldsymbol{B}}^{\boldsymbol{V}}$ and $\boldsymbol{P}_{\boldsymbol{B}}$ are given in equations (3)-(6), respectively.

One can show that for large $N$,

$$
P_{H_{1}}\left(-\kappa_{1} \log \lambda_{1}>c\right)=P\left(\chi_{f_{1}}^{2}>c\right),
$$

where

$$
\kappa_{1}=n-2\left(\frac{2 p^{2}+p+2}{12 p}-\frac{m(p-q)}{2 p}+\frac{q m(p-q)(p+m)}{2 p\left(p^{2}+p-2\right)}\right)
$$

and $f_{1}=\frac{p(p+1)}{2}-1$. See Srivastava and Carter (1977) for more details

\subsection{Likelihood Ratio Test for sphericity based on the unweighted estimator of $\xi$}

Using the estimator for $\boldsymbol{\Sigma}$ based on the unweighted estimator for $\boldsymbol{\xi}$, i.e., the estimator for the covariance matrix given in (9) we can propose another LR test statistic. This will be a modified LRT and not exact since it is not based on the distribution of the estimator. The test statistic is given by

$$
\lambda_{2}=\frac{\left|\boldsymbol{V}+\left(\boldsymbol{I}-\boldsymbol{P}_{\boldsymbol{B}}\right) \boldsymbol{V}_{1}\left(\boldsymbol{I}-\boldsymbol{P}_{\boldsymbol{B}}\right)^{\prime}\right|}{\left(\frac{\operatorname{tr} \boldsymbol{V}+\operatorname{tr}\left(\boldsymbol{I}-\boldsymbol{P}_{\boldsymbol{B}}\right) \boldsymbol{V}_{1}}{p}\right)^{p}} .
$$

It may be noted that under the hypothesis $H_{1}, \boldsymbol{V}=\sigma^{2} \boldsymbol{I}+n^{-1 / 2} \boldsymbol{U}$, where $\boldsymbol{U}=\left(u_{i j}\right)$ and $u_{i j}=O_{p}(1)$. Hence, under $H_{1}, \boldsymbol{P}_{\boldsymbol{B}}^{\boldsymbol{V}}=\boldsymbol{P}_{\boldsymbol{B}}+n^{-1 / 2} O_{p}(\boldsymbol{U})$. Thus, $\lambda_{1}$ and $\lambda_{2}$ have the same asymptotic distribution.

\subsection{LRT for sphericity based only on the matrix $V$}

We can also derive a LRT using the fact that the sum of squares matrix is Wishart distributed as $\boldsymbol{V}=\boldsymbol{X}\left(\boldsymbol{I}_{N}-\boldsymbol{P}_{\boldsymbol{A}^{\prime}}\right) \boldsymbol{X}^{\prime} \sim W_{p}(\boldsymbol{\Sigma}, n)$. This test 
statistic will be the same as under a linear model assumption and is given by

$$
\lambda_{3}=\frac{|\boldsymbol{V}|}{\left(\frac{\operatorname{tr} \boldsymbol{V}}{p}\right)^{p}} .
$$

One can show that for large $N$,

$$
P_{H_{1}}\left(-\kappa_{3} \log \lambda_{3}>c\right)=P\left(\chi_{f_{1}}^{2}>c\right),
$$

where $\kappa_{3}=n-\frac{2 p^{2}+p+2}{6 p}$. See Srivastava and Khatri (1979) Chapter 7.4 for more details.

\subsection{Test for sphericity based on a measure of sphericity using $\operatorname{tr} \boldsymbol{V}$ and $\operatorname{tr} \boldsymbol{V}^{2}$}

Following Srivastava (2005) we will give a test statistic based on $\operatorname{tr} \boldsymbol{V}$ and $\operatorname{tr} \boldsymbol{V}^{2}$. Let $a_{1}$ and $a_{2}$ be defined as

$$
a_{1}=\frac{\operatorname{tr} \Sigma}{p} \quad \text { and } \quad a_{2}=\frac{\operatorname{tr} \Sigma^{2}}{p},
$$

and let $\sigma_{i}, i=1, \ldots, p$ be the eigenvalues of $\boldsymbol{\Sigma}$. From the Cauchy-Schwarz inequality, it follows that

$$
a_{1}^{2}=\left(\frac{\sum_{i=1}^{p} \sigma_{i}}{p}\right)^{2} \leq \frac{\sum_{i=1}^{p} \sigma_{i}^{2}}{p}=a_{2},
$$

with equality if and only if $\sigma_{1}=\ldots=\sigma_{p}=\sigma^{2}$, i.e., if and only if $\boldsymbol{\Sigma}=\sigma^{2} \boldsymbol{I}_{p}$. Srivastava (2005) defined a measure of sphericity given by

$$
\lambda_{4}=\frac{a_{2}}{a_{1}^{2}}-1
$$

which is non-negative and takes the value 0 if and only if $\boldsymbol{\Sigma}=\sigma^{2} \boldsymbol{I}_{p}$. Srivastava (2005) proposed a test based on unbiased and consistent estimators of $a_{1}$ and $a_{2}$, given as

$$
\begin{aligned}
& \hat{a}_{1}=\frac{\operatorname{tr} \boldsymbol{V}}{n p}, \\
& \hat{a}_{2}=\frac{1}{p(n-1)(n+2)}\left(\operatorname{tr} \boldsymbol{V}^{2}-\frac{1}{n}(\operatorname{tr} \boldsymbol{V})^{2}\right),
\end{aligned}
$$


respectively. Furthermore, the asymptotic distribution for $\hat{a}_{1}$ is given by Srivastava (2005) and using this we can get an unbiased estimator of $a_{1}^{2}$ as

$$
\hat{a}_{1}^{2}-2 \frac{\hat{a}_{2}}{n p}
$$

since

$$
\mathrm{E}\left(\hat{a}_{1}^{2}\right)=\operatorname{var}\left(\hat{a}_{1}\right)+\left(\mathrm{E}\left(\hat{a}_{1}\right)\right)^{2}=2 \frac{a_{2}}{n p}+a_{1}^{2} .
$$

Hence, the test statistic that we propose for sphericity is

$$
\hat{\lambda}_{4}=\frac{\hat{a}_{2}}{\hat{a}_{1}^{2}-2 \frac{\hat{a}_{2}}{n p}}-1 .
$$

Under the null hypothesis that $\boldsymbol{\Sigma}=\sigma^{2} \boldsymbol{I}_{p}$, and $n=\mathcal{O}\left(p^{\delta}\right), \delta>1 / 2$, asymptotically as $(n, p) \rightarrow \infty$

$$
\frac{n}{2} \hat{\lambda}_{4} \sim N(0,1)
$$

Note that it is a one-sided test for testing the hypothesis that $\lambda_{4}=0$ vs. $\lambda_{4}>0$. Also, note that the test statistic based on $\hat{\lambda}_{4}$ can be performed for all values of $n$ and $p$ as opposed to the test based on the likelihood ratio test which requires that $n \geq p$.

\section{$5 \quad$ Testing intraclass covariance structure}

In this section we consider the hypothesis

$$
H_{2}: \boldsymbol{\Sigma}=\boldsymbol{\Sigma}_{I C} \equiv \sigma^{2}\left((1-\rho) \boldsymbol{I}_{p}+\rho \mathbf{1 1}^{\prime}\right) \quad \text { vs. } \quad A_{2}: \boldsymbol{\Sigma}>0,
$$

with $-\frac{1}{p-1}<\rho<1$. This covariance structure $\boldsymbol{\Sigma}_{I C}$ is called intraclass covariance structure. Other names are uniform or complete symmetry. We will give four different test statistics with corresponding asymptotic null distribution. In Section 6 we will compare the performance of these test.

\subsection{Standard Likelihood Ratio Test for intraclass co- variance structure}

The standard LRT testing the hypothesis $H_{2}$ is based on the MLEs, (1) and (2). This test should not be preferred, since it is not based on a unbiased 
estimator of $\boldsymbol{\Sigma}$. Anyway, the standard LRT is given by Khatri (1973) as

$$
\gamma_{1}=\frac{\left|\boldsymbol{V}+\left(\boldsymbol{I}-\boldsymbol{P}_{\boldsymbol{B}}^{\boldsymbol{V}}\right) \boldsymbol{V}_{1}\left(\boldsymbol{I}-\boldsymbol{P}_{\boldsymbol{B}}^{\boldsymbol{V}}\right)^{\prime}\right|}{\left(\frac{\mathbf{1}^{\prime} \boldsymbol{V} \mathbf{1}}{p}\right)\left(\frac{\operatorname{tr}\left(\boldsymbol{I}-\boldsymbol{P}_{\mathbf{1}}\right) \boldsymbol{V}+\operatorname{tr}\left(\boldsymbol{I}-\boldsymbol{P}_{\boldsymbol{B}}\right) \boldsymbol{V}_{1}}{p-1}\right)^{p-1}} .
$$

As usual, one can show that for large $N$ we have

$$
P_{H_{2}}\left(-n \log \gamma_{1}>c\right)=P\left(\chi_{f_{2}}^{2}>c\right)
$$

where $f_{2}=\frac{p(p+1)}{2}-2$.

\subsection{Likelihood Ratio Test for intraclass covariance struc- ture based on the unweighted estimator of $\xi$}

Again we can use the estimator of $\boldsymbol{\Sigma}$ based on the unweighted estimator for $\boldsymbol{\xi}$, i.e., the estimator for the covariance matrix given in (9). The modified LRT statistic is given by

$$
\gamma_{2}=\frac{\left|\boldsymbol{V}+\left(\boldsymbol{I}-\boldsymbol{P}_{\boldsymbol{B}}\right) \boldsymbol{V}_{1}\left(\boldsymbol{I}-\boldsymbol{P}_{\boldsymbol{B}}\right)^{\prime}\right|}{\left(\frac{\mathbf{1}^{\prime} \boldsymbol{V} \mathbf{1}}{p}\right)\left(\frac{\operatorname{tr}\left(\boldsymbol{I}-\boldsymbol{P}_{\mathbf{1}}\right) \boldsymbol{V}+\operatorname{tr}\left(\boldsymbol{I}-\boldsymbol{P}_{\boldsymbol{B}}\right) \boldsymbol{V}_{1}}{p-1}\right)^{p-1}} .
$$

The difference between the LRT $\gamma_{1}$, given in (14), and the modified LRT $\gamma_{2}$ is only in the inner product defined by $\boldsymbol{V}$ in the projector $\boldsymbol{P}_{\boldsymbol{B}}^{\boldsymbol{V}}$ in the numerator. Again, as discussed earlier in Section 4.2, this inner product will not change the asymptotic distribution. Hence, the asymptotic distribution for the modified LRT statistic $\gamma_{2}$ is the same as for the LRT statistic $\gamma_{1}$ and is given in (15).

\subsection{LRT for intraclass covariance structure based only on the matrix $V$}

Again, we can derive a LRT using the fact that $\boldsymbol{V} \sim W_{p}(\boldsymbol{\Sigma}, n)$. This test statistic will be the same as under a linear model assumption and is given by

$$
\gamma_{3}=\frac{|\boldsymbol{V}|}{\left(\frac{\mathbf{1}^{\prime} \boldsymbol{V} \mathbf{1}}{p}\right)\left(\frac{p \operatorname{tr} \boldsymbol{V}-\mathbf{1}^{\prime} \boldsymbol{V} \mathbf{1}}{p(p-1)}\right)^{p-1}}
$$

For large $N$, Box $(1949,1950)$ has shown that

$$
P_{H_{2}}\left(-\nu_{3} \log \gamma_{3}>c\right)=P\left(\chi_{f_{2}}^{2}>c\right),
$$

where $\nu_{3}=n-\frac{p(p+1)^{2}(2 p-3)}{6(p-1)\left(p^{2}+p-4\right)}$. 


\subsection{Test for intraclass covariance structure based on a measure of sphericity}

Let the matrix $\boldsymbol{Q}$ be an orthogonal matrix of order $p$ and let the first column be a normalized column of ones, i.e.,

$$
\boldsymbol{Q}=\left(\begin{array}{ll}
p^{-1 / 2} \mathbf{1}_{p} & \left.\boldsymbol{Q}_{2}\right): p \times p
\end{array}\right.
$$

Given the one-to-one orthogonal transformation $\boldsymbol{X}^{*}=\boldsymbol{Q}^{\prime} \boldsymbol{X}$ we have the following equivalent model

$$
\boldsymbol{X}^{*} \sim N_{p, N}\left(\boldsymbol{B}^{*} \boldsymbol{\xi} \boldsymbol{A}, \boldsymbol{\Sigma}^{*}, \boldsymbol{I}_{N}\right),
$$

where

$$
\begin{aligned}
& \boldsymbol{B}^{*}=\boldsymbol{Q}^{\prime} \boldsymbol{B} \\
& \boldsymbol{\Sigma}^{*}=\boldsymbol{Q}^{\prime} \boldsymbol{\Sigma} \boldsymbol{Q}=\left(\begin{array}{cc}
\sigma_{11}^{*} & \boldsymbol{\sigma}_{12}^{*} \\
\boldsymbol{\sigma}_{21}^{*} & \boldsymbol{\Sigma}_{22}^{*}
\end{array}\right),
\end{aligned}
$$

and $\boldsymbol{\sigma}_{12}^{*}=\left(\boldsymbol{\sigma}_{21}^{*}\right)^{\prime}: 1 \times(p-1), \boldsymbol{\Sigma}_{22}^{*}:(p-1) \times(p-1)$. However, under the null hypothesis $H_{2}$ we have

$$
\boldsymbol{\Sigma}^{*}=\boldsymbol{Q}^{\prime} \boldsymbol{\Sigma}_{I C} \boldsymbol{Q}=\left(\begin{array}{cc}
\sigma^{2}(1+(p-1) \rho) & \mathbf{0}^{\prime} \\
\mathbf{0} & \sigma^{2}(1-\rho) \boldsymbol{I}_{p-1}
\end{array}\right)
$$

Instead of testing the hypothesis given in (13), we will test the hypothesis

$$
H_{3}: \boldsymbol{\Sigma}_{22}^{*}=\tilde{\sigma}^{2} \boldsymbol{I}_{p-1} \quad \text { vs. } \quad A_{3}: \boldsymbol{\Sigma}_{22}^{*}>0,
$$

for some $\tilde{\sigma}^{2}$, even if we loose some information due to the fact that we do not test if $\boldsymbol{\sigma}_{12}^{*}=\mathbf{0}^{\prime}$. Hypothesis $H_{3}$ is tested using the same procedure as for testing sphericity in Subsection 4.4.

\section{Compare the performance}

To compare the performance for the different tests we can compute the attained significance level (ASL) and the empirical power. Let $c$ be the critical value from the distribution considered for the test statistics. With 10,000 simulated replications under the null hypothesis, the ASL is computed as

$$
\widehat{\alpha}=\frac{\left(\# \text { of } t_{H} \geq c\right)}{(\# \text { simulated replications })}
$$


where $t_{H}$ is the value of the test statistic derived from the simulated data under the null hypothesis. We set the nominal significance level to $\alpha=5 \%$.

For the simulations let

$$
\boldsymbol{B}_{1}=\left(\begin{array}{cccc}
1 & 1 & \cdots & 1 \\
1 & 2 & \cdots & p
\end{array}\right)^{\prime}
$$

or $\boldsymbol{B}_{2}=\left(b_{i j}\right)$, where $b_{i j} \sim U(0,2), i=1, \ldots, p, j=1,2$ and

$$
\boldsymbol{A}=\left(\begin{array}{cc}
\mathbf{1}_{N_{1}}^{\prime} & \mathbf{0}_{N_{2}}^{\prime} \\
\mathbf{0}_{N_{1}}^{\prime} & \mathbf{1}_{N_{2}}^{\prime}
\end{array}\right)
$$

i.e., with $q=2$ and $m=2$. For simplicity we will choose $N$ even and $N_{1}=N_{2}=N / 2$.

For the power simulations let $\boldsymbol{D}=\operatorname{diag}\left(\sigma_{1}, \ldots, \sigma_{p}\right)$ and define $\sigma_{i}=$ $\sqrt{U[0.9,1.1]}$, for $i=1, \ldots, p$, Also, let $\boldsymbol{R}=\left(\rho_{i j}\right)$, where $\rho_{i j}=(-1)^{i+j}\left(\frac{r}{\log p}\right)^{|i-j|^{f}}$. Under the alternatives we will assume the covariance matrix

$$
\boldsymbol{\Sigma}_{A}=\boldsymbol{D} \boldsymbol{R} \boldsymbol{D}, \quad \text { with } r=0.5 \text { and } f=0.1
$$

We can compute the empirical power using two different critical values. We can either use the critical value $c$ from the asymptotic distribution, or we can use the estimated critical value $\widehat{c}$ calculated from the simulated data under the null hypothesis, i.e., the critical value calculated from the empirical null distribution. We will use the estimated critical value since the ASL is greatly affected for some tests. The empirical power is calculated from 10, 000 new replications simulated under the alternative hypothesis when $\boldsymbol{\Sigma}=\boldsymbol{\Sigma}_{A}$. Let $t_{A}$ be the value of the test statistic derived from the simulated data under the alternative hypothesis. The empirical power is given as

$$
\widehat{\beta}=\frac{\left(\# \text { of } t_{A} \geq \widehat{c}\right)}{(\# \text { simulated replications })} .
$$




\begin{tabular}{|c|c|c|c|c|c|c|c|c|c|}
\hline \multirow[b]{3}{*}{$N$} & \multirow[b]{3}{*}{$p$} & \multicolumn{8}{|c|}{$\overline{B_{1}}$} \\
\hline & & \multicolumn{4}{|c|}{ ASL $\widehat{\alpha}(\alpha=5 \%)$} & \multicolumn{4}{|c|}{ Power $\widehat{\beta}$} \\
\hline & & $\lambda_{1}$ & $\lambda_{2}$ & $\lambda_{3}$ & $\lambda_{4}$ & $\lambda_{1}$ & $\lambda_{2}$ & $\lambda_{3}$ & $\lambda_{4}$ \\
\hline 8 & 4 & 7.77 & 1.82 & 6.69 & 6.14 & 15.42 & 18.50 & 12.79 & 20.26 \\
\hline 8 & 6 & 33.67 & 2.96 & 25.01 & 5.62 & 8.05 & 12.03 & 7.57 & 19.80 \\
\hline 8 & 16 & - & - & - & 5.49 & - & - & - & 20.47 \\
\hline 2 & 3 & 5.40 & 3.37 & 5.20 & 6.21 & 41.12 & 43.72 & 33.25 & 41.22 \\
\hline 12 & 6 & 8.04 & 2.82 & 6.81 & 6.15 & 21.42 & 25.95 & 18.14 & 33.91 \\
\hline 12 & 9 & 27.98 & 5.44 & 22.43 & 5.67 & 12.05 & 17.65 & 10.67 & 34.28 \\
\hline 12 & 24 & - & - & - & 5.42 & - & - & - & 34.01 \\
\hline 20 & 5 & 4.86 & 3.29 & 4.62 & 6.02 & 52.47 & 54.59 & 46.10 & 59.21 \\
\hline 20 & 10 & 8.56 & 4.56 & 7.55 & 6.26 & 33.34 & 34.99 & 29.97 & 56.67 \\
\hline 20 & 15 & 26.35 & 11.46 & 25.52 & 5.82 & 21.37 & 24.58 & 19.10 & 57.36 \\
\hline 20 & 40 & - & - & - & 5.36 & - & - & - & 58.66 \\
\hline 60 & 15 & 5.61 & 4.80 & 5.48 & 5.57 & 92.52 & 92.62 & 91.34 & 98.79 \\
\hline 60 & 30 & 11.21 & 9.24 & 11.41 & 6.04 & 81.59 & 82.08 & 79.68 & 98.91 \\
\hline 60 & 45 & 57.13 & 50.01 & 61.00 & 5.91 & 64.33 & 65.40 & 61.24 & 99.38 \\
\hline 60 & 120 & - & - & - & 4.96 & - & - & - & 99.40 \\
\hline 100 & 25 & 5.92 & 5.28 & 5.66 & 5.61 & 99.63 & 99.64 & 99.55 & 99.98 \\
\hline 100 & 50 & 15.92 & 14.31 & 15.96 & 5.29 & 97.83 & 97.81 & 97.50 & 100.00 \\
\hline 100 & 75 & 88.25 & 86.02 & 90.52 & 5.21 & 89.29 & 89.58 & 87.80 & 100.00 \\
\hline 100 & 200 & - & - & - & 5.32 & - & - & - & 100.00 \\
\hline 200 & 50 & 6.69 & 6.43 & 6.78 & 5.98 & 100.00 & 100.00 & 100.00 & 100.00 \\
\hline 200 & 100 & 32.01 & 30.91 & 32.91 & 5.35 & 100.00 & 100.00 & 100.00 & 100.00 \\
\hline 200 & 150 & 1.40 & 1.54 & 32.26 & 5.26 & 93.65 & 95.06 & 99.82 & 100.00 \\
\hline \multirow[t]{2}{*}{200} & 400 & - & - & - & 4.95 & - & - & - & 100.00 \\
\hline & & \multicolumn{8}{|c|}{$\overline{\boldsymbol{B}_{2}}$} \\
\hline & & \multicolumn{4}{|c|}{ ASL $\widehat{\alpha}(\alpha=5 \%)$} & \multicolumn{4}{|c|}{ Power $\widehat{\beta}$} \\
\hline$N$ & $p$ & $\lambda_{1}$ & $\lambda_{2}$ & $\lambda_{3}$ & $\lambda_{4}$ & $\lambda_{1}$ & $\lambda_{2}$ & $\lambda_{3}$ & $\lambda_{4}$ \\
\hline 8 & 4 & 7.40 & 1.82 & 6.36 & 5.72 & 12.75 & 13.51 & 12.80 & 21.76 \\
\hline 8 & 6 & 32.89 & 2.48 & 24.23 & 5.88 & 6.30 & 9.89 & 6.61 & 19.32 \\
\hline 8 & 16 & - & - & - & 5.90 & - & - & - & 19.70 \\
\hline 12 & 3 & 5.28 & 3.47 & 5.18 & 5.81 & 37.05 & 36.38 & 32.95 & 41.70 \\
\hline 12 & 6 & 8.46 & 2.86 & 6.64 & 5.60 & 20.31 & 24.54 & 18.64 & 33.72 \\
\hline 12 & 9 & 27.69 & 5.10 & 22.17 & 5.68 & 12.04 & 17.24 & 10.22 & 32.90 \\
\hline 12 & 24 & - & - & - & 5.72 & - & - & - & 33.00 \\
\hline 20 & 5 & 5.50 & 3.77 & 5.38 & 6.78 & 46.05 & 45.44 & 43.68 & 57.93 \\
\hline 20 & 10 & 8.13 & 4.14 & 7.26 & 6.09 & 33.42 & 35.17 & 30.24 & 56.93 \\
\hline 20 & 15 & 26.74 & 11.86 & 25.33 & 6.06 & 19.01 & 23.25 & 17.17 & 56.48 \\
\hline 20 & 40 & - & - & - & 5.64 & - & - & - & 58.09 \\
\hline 60 & 15 & 5.46 & 4.58 & 5.54 & 6.18 & 91.89 & 91.97 & 90.92 & 98.45 \\
\hline 60 & 30 & 11.01 & 9.03 & 10.93 & 5.48 & 83.41 & 83.70 & 80.27 & 99.09 \\
\hline 60 & 45 & 57.57 & 50.46 & 61.58 & 5.48 & 62.09 & 63.20 & 59.27 & 99.13 \\
\hline 60 & 120 & - & - & - & 5.22 & - & - & - & 99.52 \\
\hline 100 & 25 & 5.84 & 5.38 & 5.68 & 5.97 & 99.53 & 99.51 & 99.49 & 99.99 \\
\hline 100 & 50 & 15.05 & 13.66 & 15.52 & 5.22 & 97.86 & 97.85 & 97.63 & 100.00 \\
\hline 100 & 75 & 88.12 & 85.88 & 90.29 & 5.73 & 89.37 & 89.55 & 88.05 & 100.00 \\
\hline 100 & 200 & - & - & - & 5.15 & - & - & - & 100.00 \\
\hline 200 & 50 & 6.61 & 6.35 & 6.56 & 5.26 & 100.00 & 100.00 & 100.00 & 100.00 \\
\hline 200 & 100 & 31.83 & 30.76 & 32.46 & 4.83 & 100.00 & 100.00 & 100.00 & 100.00 \\
\hline 200 & 150 & 1.79 & 1.64 & 32.93 & 5.12 & 86.95 & 86.06 & 99.67 & 100.00 \\
\hline 200 & 400 & - & - & - & 5.38 & - & - & - & 100.00 \\
\hline
\end{tabular}

Table 1: Sphericity - ASL and Power (\%) 


\begin{tabular}{|c|c|c|c|c|c|c|c|c|c|}
\hline \multirow[b]{3}{*}{$N$} & \multirow[b]{3}{*}{$p$} & \multicolumn{8}{|c|}{$\overline{\boldsymbol{B}_{1}}$} \\
\hline & & \multicolumn{4}{|c|}{ ASL $\widehat{\alpha}(\alpha=5 \%)$} & \multicolumn{4}{|c|}{ Power $\widehat{\beta}$} \\
\hline & & $\gamma_{1}$ & $\gamma_{2}$ & $\gamma_{3}$ & $\gamma_{4}$ & $\gamma_{1}$ & $\gamma_{2}$ & $\gamma_{3}$ & $\gamma_{4}$ \\
\hline 12 & 3 & 8.97 & 6.22 & 5.10 & 5.95 & 35.05 & 39.18 & 28.61 & 29.53 \\
\hline 12 & 4 & 10.81 & 5.84 & 5.47 & 6.41 & 22.09 & 27.88 & 20.33 & 33.57 \\
\hline 12 & 6 & 19.02 & 8.47 & 6.56 & 5.90 & 18.25 & 13.23 & 17.61 & 33.88 \\
\hline 12 & 24 & - & - & - & 5.83 & - & - & - & 33.98 \\
\hline 24 & 6 & 8.87 & 6.46 & 5.39 & 6.94 & 50.97 & 44.08 & 47.76 & 68.24 \\
\hline 24 & 8 & 11.85 & 8.32 & 5.27 & 6.09 & 47.64 & 30.74 & 43.72 & 67.67 \\
\hline 24 & 12 & 30.59 & 22.71 & 7.76 & 6.17 & 37.23 & 13.34 & 34.49 & 66.86 \\
\hline 24 & 48 & - & - & - & 5.24 & - & - & - & 69.47 \\
\hline 48 & 12 & 11.65 & 9.87 & 5.20 & 6.12 & 84.31 & 45.72 & 82.11 & 95.72 \\
\hline 48 & 16 & 22.68 & 19.70 & 5.93 & 6.04 & 80.07 & 30.58 & 78.05 & 95.48 \\
\hline 48 & 24 & 71.61 & 67.00 & 9.54 & 5.70 & 71.27 & 14.73 & 67.05 & 96.25 \\
\hline 48 & 96 & - & - & - & 5.35 & - & - & - & 97.32 \\
\hline 96 & 24 & 24.89 & 23.35 & 5.66 & 5.58 & 99.46 & 48.53 & 99.26 & 100.00 \\
\hline 96 & 32 & 58.36 & 56.26 & 7.06 & 5.84 & 99.05 & 35.17 & 98.81 & 100.00 \\
\hline 96 & 48 & 99.81 & 99.74 & 15.22 & 5.52 & 97.15 & 21.16 & 96.53 & 100.00 \\
\hline 96 & 192 & - & - & - & 5.10 & - & - & - & 100.00 \\
\hline 192 & 48 & 65.51 & 64.66 & 6.17 & 5.60 & 100.00 & 61.89 & 100.00 & 100.00 \\
\hline 192 & 64 & 98.38 & 98.29 & 8.95 & 5.19 & 100.00 & 54.83 & 100.00 & 100.00 \\
\hline 192 & 96 & 100.00 & 100.00 & 30.74 & 5.12 & 100.00 & 47.28 & 100.00 & 100.00 \\
\hline \multirow[t]{3}{*}{192} & 384 & - & - & - & 4.95 & - & - & - & 100.00 \\
\hline & & \multicolumn{8}{|c|}{$\boldsymbol{B}_{2}$} \\
\hline & & \multicolumn{4}{|c|}{$\mathrm{ASL} \widehat{\alpha}(\alpha=5 \%)$} & \multicolumn{4}{|c|}{ Power $\widehat{\beta}$} \\
\hline$N$ & $p$ & $\gamma_{1}$ & $\gamma_{2}$ & $\gamma_{3}$ & $\gamma_{4}$ & $\gamma_{1}$ & $\gamma_{2}$ & $\gamma_{3}$ & $\gamma_{4}$ \\
\hline 12 & 3 & 11.73 & 6.85 & 5.25 & 6.05 & 28.85 & 39.88 & 30.10 & 28.64 \\
\hline 12 & 4 & 11.31 & 5.91 & 5.24 & 5.85 & 19.87 & 32.14 & 20.08 & 34.41 \\
\hline 12 & 6 & 24.22 & 9.36 & 6.95 & 5.99 & 13.18 & 27.61 & 16.61 & 34.08 \\
\hline 12 & 24 & - & - & - & 5.59 & - & - & - & 33.18 \\
\hline 24 & 6 & 10.09 & 6.73 & 5.32 & 6.62 & 47.71 & 53.47 & 47.04 & 67.37 \\
\hline 24 & 8 & 14.73 & 8.97 & 5.68 & 6.43 & 41.91 & 52.15 & 43.11 & 66.92 \\
\hline 24 & 12 & 39.53 & 24.40 & 7.85 & 6.48 & 29.99 & 43.31 & 34.56 & 66.36 \\
\hline 24 & 48 & - & - & - & 5.13 & - & - & - & 70.24 \\
\hline 48 & 12 & 18.50 & 11.24 & 5.47 & 6.19 & 76.73 & 80.28 & 81.38 & 95.52 \\
\hline 48 & 16 & 29.94 & 20.73 & 5.98 & 6.45 & 71.94 & 75.61 & 77.11 & 95.41 \\
\hline 48 & 24 & 78.64 & 69.50 & 9.89 & 5.80 & 62.93 & 67.70 & 68.18 & 96.08 \\
\hline 48 & 96 & - & - & - & 5.29 & - & - & - & 97.29 \\
\hline 96 & 24 & 32.85 & 25.38 & 5.83 & 6.03 & 99.06 & 98.33 & 99.32 & 99.99 \\
\hline 96 & 32 & 67.01 & 57.43 & 6.78 & 5.57 & 98.13 & 97.31 & 98.81 & 100.00 \\
\hline 96 & 48 & 99.87 & 99.82 & 15.31 & 5.53 & 95.29 & 93.12 & 96.19 & 100.00 \\
\hline 96 & 192 & - & - & - & 5.08 & - & - & - & 100.00 \\
\hline 192 & 48 & 73.54 & 66.55 & 6.34 & 5.58 & 100.00 & 100.00 & 100.00 & 100.00 \\
\hline 192 & 64 & 98.84 & 98.27 & 8.69 & 5.22 & 100.00 & 100.00 & 100.00 & 100.00 \\
\hline 192 & 96 & 100.00 & 100.00 & 31.42 & 5.21 & 99.99 & 99.98 & 99.99 & 100.00 \\
\hline 192 & 384 & - & - & - & 4.90 & - & - & - & 100.00 \\
\hline
\end{tabular}

Table 2: Intraclass covariance structure - ASL and Power (\%) 


\section{Conclusion}

In Table 1 we see that when testing sphericity, $\lambda_{4}$ seems to be the best. For small $p$ compared to $N$ there is no really difference, but when $p$ is larger, $\lambda_{4}$ is definitely better with controlled ASL and good power. Observe also that the test statistic $\lambda_{4}$ works fine for high dimensions, i.e., when $p>N$. One can note the small ASL for $\lambda_{1}$ and $\lambda_{2}$ for the case $N=200, p=150$. This is probably due to numerical problems.

For testing the intraclass covariance structure we see, in Table 2, that test statistic $\gamma_{4}$ controls the ASL better then the others. Also the power is better with $\gamma_{4}$ for most of the cases. Note also that $\gamma_{3}$ seems to behave pretty well with controlled ASL and good power. Observe again that the test statistic $\gamma_{4}$ works fine for high dimensions.

\section{References}

Box, G. E. (1949). A general distribution theory for a class of likelihood criteria. Biometrika, 36(3/4):317-346.

Box, G. E. (1950). Problems in the analysis of growth and wear curves. Biometrics, 6(4):362-389.

Chinchilli, V. M. and Walter Jr, H. C. (1984). A likelihood ratio test for a patterned covariance matrix in a multivariate Growth Curve model. Biometrics, 40:151-156.

Khatri, C. G. (1966). A note on a MANOVA model applied to problems in Growth Curve. Annals of the Institute of Statistical Mathematics, 18:7586.

Khatri, C. G. (1973). Testing some covariance structures under a Growth Curve model. Journal of Multivariate Analysis, 3(1):102-116.

Klein, D. and Žežula, I. (2010). Orthogonal decompositions in Growth Curve models. Acta et Commentationes Universitatis Tartuensis de Mathematica, $14: 35-44$.

Kollo, T. and von Rosen, D. (2005). Advanced Multivariate Statistics with Matrices. Springer, Dordrecht, The Netherlands.

Kshirsagar, A. M. and Smith, W. B. (1995). Growth Curves. Marcel Dekker, New York. 
Lee, J. C. (1988). Prediction and estimation of growth curves with special covariance structures. Journal of the American Statistical Association, $83: 432-440$.

Potthoff, R. F. and Roy, S. (1964). A generalized multivariate analysis of variance model useful especially for growth curve problems. Biometrika, $51(3 / 4): 313-326$.

Rao, C. R. (1965). The theory of least squares when the parameters are stochastic and its application to the analysis of growth curves. Biometrika, 52(3/4):447-458.

Rao, C. R. (1967). Least squares theory using an estimated dispersion matrix and its application to measurement of signals. In Proceedings of the Fifth Berkeley Symposium on Mathematial Statistics and Probability. Vol I (L. M. LeCam and J. Neyman, eds.). University of California Press, Berkeley, pages 355-372.

Rao, C. R. (1975). Simultaneous estimation of parameters in different linear models and applications to biometric problems. Biometrics, 31(2):545-554.

Reinsel, G. (1982). Multivariate repeated measurement or Growth Curve models with multivariate random-effects covariance structure. Journal of the American Statistical Association, 77(377):190-195.

Reinsel, G. (1984). Estimation and prediction in a multivariate random effects generalized linear model. Journal of the American Statistical Association, 79(386):406-414.

Srivastava, M. S. (2005). Some tests concerning the covariance matrix in high dimensional data. Journal of the Japan Statistical Society, 35(2):251-272.

Srivastava, M. S. and Carter, E. M. (1977). Asymptotic non-null distribution of a likelihood ratio criterion for sphericity in the growth curve model. Sankhyā: The Indian Journal of Statistics, Series B, pages 160-165.

Srivastava, M. S. and Khatri, C. G. (1979). An Introduction to Multivariate Statistics. Elsvier North Holland, New York, USA.

Srivastava, M. S. and Singull, M. (2012). Profile analysis with random-effects covariance structure. Journal of the Japan Statistical Society, 42(2):145164. 
Srivastava, M. S. and Singull, M. (2015). Test for the mean matrix in a Growth Curve model for high dimensions. Accepted for publication in Communications in statistics - Theory and methods, pages 1-26.

Srivastava, M. S. and von Rosen, D. (1999). Growth Curve model. In Multivariate Analysis, Design of Experiments, and Survey Sampling, Textbooks Monogr. 159 Dekker, New York, pages 547-578.

von Rosen, D. (1991). Moments of maximum likelihood estimators in the Growth Curve model. Statistics, 22(1):111-131.

Ware, J. H. (1985). Linear models for the analysis of longitudinal studies linear models for the analysis of longitudinal studies. The American Statistician, 39(2):95-101.

Wilks, S. S. (1946). Sample criteria for testing equality of means, equality of variances, and equality of covariances in a normal multivariate distribution. Annals of Mathematical Statistics, 17:257-281.

Ye, R.-D. and Wang, S.-G. (2009). Estimating parameters in extended Growth Curve models with special covariance structures. Journal of Statistical Planning and Inference, 139(8):2746-2756.

Yokoyama, T. (1995). Statistical inference on some mixed MANOVAGMANOVA models with random effects. Hiroshima Math. J., 25(3):441474.

Yokoyama, T. (1996). Extended Growth Curve models with random-effects covariance structures. Communications in statistics - Theory and methods, 25(3):571-584.

Yokoyama, T. (1997). Tests for a family of random-effects covariance structures in a multivariate Growth Curve model. Journal of Statistical Planning and Inference, 65(2):281-292.

Žežula, I. (1993). Covariance components estimation in the Growth Curve model. Statistics: A Journal of Theoretical and Applied Statistics, 24(4):321-330.

Žežula, I. (2006). Special variance structures in the Growth Curve model. Journal of Multivariate Analysis, 97:606-618. 
Authors:

Muni S. Srivastava

Department of Statistics, University of Toronto,

100 St. George Street, Toronto, Canada, M5S 3G3

E-mail: srivasta@utstat.toronto.edu

Martin Singull (corresponding author)

Department of Mathematics, Linköping University,

SE-581 83 Linköping, Sweden

E-mail: martin.singull@liu.se 